\title{
Benefícios do uso de melatonina no tratamento de pessoas com distúrbios do sono
}

\author{
Benefits of using melatonin to treat people with sleep disorders
}

\author{
Wellington Oliveira de Carvalho", Juliana Azevedo da Paixão ${ }^{\circ}$
}

Universidade Salvador, Salvador, Bahia, Brasil. *Autor para correspondência. E-mal: wncarvalho@hotmail.com

\begin{abstract}
Resumo: Introdução: Sabe-se que a qualidade do sono interfere de forma significativa na saúde física, mental e social das pessoas. Entretanto, por ocasião da agitação do dia a dia, com os compromissos, trabalhos e tarefas cada vez maiores, além da pré-disposição genética e outros fatores, o sono tem sido comprometido cada vez mais. Na tentativa de suprir essa necessidade, muitas pessoas têm recorrido ao uso de medicamentos ansiolíticos para ajudar a dormir, sem considerar os riscos e seu efeitos colaterais. Revisão: Este trabalho foi realizado através da revisão de literatura com o objetivo de avaliar os efeitos da melatonina no tratamento contra a insônia, como uma possível alternativa com menores efeitos adversos frente aos benzodiazepínicos comumente usados, em especial o clonazepam. Discussão: A melatonina é um hormônio produzido principalmente à noite pela glândula pineal e tem como principal função a indução ao sono. Alguns fatores podem diminuir a sua produção, comprometendo a qualidade do sono. Vários estudos têm mostrado que o uso da melatonina exógena contribui de forma significativa para o ajuste do ciclo circadiano, melhorando a qualidade do sono. Ademais, comparada com os benzodiazepínicos utilizados contra insônia, estudos encontrados mostram que a melatonina possui menos reações adversas, além de não causar dependência, produzindo dessa forma, impactos menores à saúde. Considerações finais: Portanto, é de suma importância que haja um olhar mais cuidadoso para o tratamento da insônia com danos mínimos à saúde, através de alternativas eficazes e com efeitos adversos diminuídos, a fim de contribuir para a qualidade de vida das pessoas. A melatonina mostra-se como uma boa opção para esse fim. Há, entretanto, a necessidade de melhorar sua acessibilidade, possibilitando a expansão da sua utilização, porém, sob orientações do uso racional.
\end{abstract}

Palavras-chave: efeitos adversos, clonazepam, insônia, melatonina, distúrbios do sono.

\begin{abstract}
Introduction: It is known that the quality of sleep significantly interferes in people's physical, mental and social health. However, during the day-to-day hustle and bust, with increasing commitments, jobs and tasks, in addition to genetic predisposition and other factors, sleep has been increasingly compromised. In an attempt to meet this need, many people have resorted to the use of anxiolytic drugs to help sleep, without considering the risks and their side effects. Review: This work was carried out through a literature review with the objective of evaluating the effects of melatonin in the treatment against insomnia, as an alternative treatment with less adverse effects compared to the commonly used benzodiazepines, especially Clonazepam. Discussion: Melatonin is a hormone produced mainly at night by the pineal gland and its main function is to induce sleep. Some factors can decrease its production, compromising the quality of sleep. Several studies have shown that the use of exogenous melatonin contributes significantly to the adjustment of the circadian cycle, improving the quality of sleep. Final remarks: Therefore, it is of utmost importance that there is a more careful look at the treatment of insomnia with less damage to health, through effective alternatives and with minimal adverse effects, in order to contribute to the quality of life of people. Melatonin proves to be a good option for this purpose. However, there is a need to improve its accessibility, allowing the expansion of its use, however, under guidelines of rational use.
\end{abstract}

Keywords: adverse effects, clonazepam, melatonin, insomnia, sleep disorders.

\section{Introdução}

Embora tenha sido considerado como uma parte passiva na vida humana, o sono possui funções fundamentais que contribuem para manutenção vital como a reparação, proteção, conservação de energia e imunológica. A sua privação interfere no bem-estar mental e físico, além de gerar grandes prejuízos sociais e interpessoais (Quinhones \& Gomes, 2011). 
Produzida através da glândula pineal, localizada nos hemisférios cerebrais, a melatonina é um hormônio endógeno que além de ter ação antioxidante, tem funções importantes como controlar os ritmos circadianos, atuar na restauração neural e promover a regulação do sono, tendo sua produção aumentada durante a noite (Xie et al., 2017).

Durante a síntese da melatonina endógena é necessário que a glândula pineal realize a captação do aminoácido triptofano presente no plasma para a conversão em 5-hidroxitriptofano, através da enzima triptofano-5-hidroxilase. A serotonina posteriormente é convertida em $\mathrm{N}$-acetil-serotonina através da enzima arilalquilamina 5-acetiltranserase, responsável pela produção de $\mathrm{N}$-acetil-5-metoxitriptamina (melatonina) que ocorre preferencialmente durante a noite pela estimulação simpática (Glanzmann et al., 2019).

A melatonina é um hormônio usado por pessoas que têm dificuldade em começar a dormir, manter o sono e também melhorar a qualidade de sono durante a noite. Alguns indivíduos são mais beneficiados com uso de melatonina como os idosos, que produzem em baixa quantidade; trabalhadores noturnos, que precisam dormir durante o dia; pessoas vespertinas, que dormem e acordam tarde; pessoas que viajam e têm problemas com fuso horário (jet lag) e pessoas com cegueira, pois não têm boa noção de luminosidade e consequentemente ocorre alteração na sua produção (Rosa \& Borja, 2016).

Embora a melatonina exógena seja comercializada livremente nos EUA e Europa, sendo considerada como suplemento, no Brasil a venda ainda é proibida, mesmo tratando-se de uma substância de baixa toxicidade, diferente de vários agentes terapêuticos utilizados como indutores do sono que são liberados para o livre comércio (Bruin, 2015). Em relação às possíveis reações adversas da melatonina, existe boa tolerabilidade e segurança, e essas estão relacionadas à sonolência diurna, dor de cabeça e raramente sintomas intestinais, podendo através do ajuste da dose, serem amenizados (Silva et al., 2020).

A insônia é um dos distúrbios do sono que atinge pessoas de várias idades em todo mundo e o seu tratamento geralmente inicia-se com diagnóstico em consulta psicológica seguida de terapia medicamentosa. A maioria dos medicamentos utilizados no tratamento de insônia tem o mesmo objetivo, porém agem em sítios diferentes e são classificados em: hipnóticos, benzodiazepínicos e anti-histamínicos (Rosa \& Borja, 2016). O período de insônia de até quatro semanas geralmente não necessita de tratamento, visto que, nesse tempo é considerada de curta duração. Se persistir após essa fase deve-se buscar investigação abrangente, pois já é classificada de alta intensidade ou estar relacionada a outros sinais e sintomas, uma vez que ela pode surgir como sintoma de uma falsa doença ou uma condição clínica distinta (Rémi et al., 2019).

Entre os benzodiazepínicos comumente utilizados para combater a insônia está o clonazepam, que tem propriedade ansiolítica e hipnótica, diminuindo o tempo de início do sono dando uma falsa sensação de sonolência, mas que pode gerar problemas a curto e longo prazo como déficit de memória e dependência. $\mathrm{O}$ clonazepam tem registro na Agencia Nacional de Vigilância Sanitária (ANVISA) para o tratamento de distúrbios epilépticos, transtornos de ansiedade e de humor. Possui sua apresentação na forma de comprimidos, comprimidos sublinguais, solução oral e pertence ao formulário terapêutico nacional no qual está sujeito a controle especial (Rosa \& Borja, 2016).

Além de ser um fármaco potente, o clonazepam possui uma meia-vida longa, por isso há preocupação com os efeitos de seu uso prolongado e potencial para abuso. É importante destacar as principais reações adversas ao clonazepam como sonolência, ataxia, perda de memória, déficit de atenção, euforia transitória, podendo causar depressão respiratória, dificuldade de coordenação motora, convulsões, delírio, psicose, depressão, além de gerar comportamentos agressivos se forem utilizados com bebidas alcóolicas (Frauger et al., 2011). Diferente dos benzodiazepínicos, a melatonina não leva ao efeito de ressaca, nem à dependência e doses diárias em quantidades pequenas em indivíduos saudáveis podem induzir ao sono, além de ser favorável ao tratamento de insônia (Nakaoka et al., 2013).

Conforme abordado, o tema deste trabalho é de grande relevância social, pois trata de um problema vivido por inúmeras pessoas que fazem uso de medicamentos controlados contra insônia e que causam vários efeitos colaterais, inclusive a dependência. Há, portanto, a necessidade de expandir mais informações sobre os benefícios do tratamento com melatonina frente aos ansiolíticos, hipnóticos e sedativos, vislumbrando uma forma menos prejudicial à saúde de tratar a insônia com efeitos adversos minimizados, principalmente em época de pandemia, onde vários fatores têm tirado o sono da população.

Portanto, através de revisão bibliográfica, o presente trabalho propôs identificar na literatura dados científicos que possam colaborar com a discussão e o entendimento sobre a melatonina no tratamento contra a insônia causada pelos distúrbios do sono e comparar os seus benefícios e efeitos adversos com os medicamentos comumente usados, em especial o clonazepam que tem como referência o Rivotril, tendo, por conseguinte, o intuito de promover uma melhor qualidade de vida dos pacientes. 


\section{Revisão}

Para a realização deste estudo foi feita uma revisão sistemática integrativa de literatura nas bases de dados Pubmed, Scientific Eletronic Libraey Online (SciELO) e Google acadêmico, utilizando os seguintes descritores: Adverse Effects, Clonazepam, Melatonin, Insomnia e Sleep Disorders através de cruzamentos e empregando o conectivo "and".

Os critérios utilizados para a seleção dos artigos pesquisados foram: abordar questões do sono; identificar os efeitos da melatonina sobre a insônia; apresentar os efeitos adversos dos benzodiazepínicos usados contra a insônia; estar publicado nos idiomas: português, inglês ou espanhol; ter sido publicado nos últimos 10 anos. Para critério de exclusão foram usados os seguintes: artigos que não relacionassem o uso da melatonina à insônia; artigos sem versão completa disponível; artigos em outros idiomas; artigos duplicados nas bases de dados e trabalhos de conclusão de curso.

A partir da busca realizada, baseada nos critérios acima mencionados, não foram encontrados artigos que estivessem relacionados a todos os descritores juntos, sendo, portanto, encontrados de forma separada ou cruzada com números menores. De forma geral foram encontrados cerca de 3.240 artigos nas bases de dados pesquisadas, porém, após critérios de exclusão e leitura criteriosa foram utilizados apenas 25 artigos para a realização deste trabalho.

\section{Discussão}

\section{A melatonina no tratamento contra a insônia}

Várias pesquisas têm sugerido que o sono e ritmos circadianos contribuem de forma relevante com a aprendizagem e a memória. O sono interrompido ou a sua privação tem impacto na função cognitiva, entretanto, sua manutenção beneficia a aprendizagem por meio de sinapse global. O comprometimento da manutenção homeostática do sono pode ocasionar em alterações clínicas importantes como transtornos psiquiátricos, incluindo depressão e esquizofrenia (Vyazovskiy et al., 2017).

Geralmente a insônia é proveniente de evento estressor, mas pode estar relacionada a algumas condições clínicas surgindo como sintoma e evoluindo para transtorno, já que a diferença entre essas condições é a dimensão do impacto clínico que ocorre no paciente. Entre as condições clínicas que podem estimular a insônia estão: asma, insuficiência cardíaca, menopausa, hipertireoidismo, gravidez e doenças do Sistema Nervoso Central (SNC), assim como o uso ou interrupção de medicamentos como benzodiazepínicos, anticonvulsivantes, antidepressivos, corticoides e algumas substâncias como cafeína e álcool (Bacelar \& Pinto Júnior, 2019). Outrossim, visto como marcador confiável para risco de insônia, o histórico familiar, principalmente materno, também tem contribuído para o desenvolvimento desse transtorno com grande potencial (Kalmbach et al., 2018).

O principal tratamento para a insônia crônica é realizado geralmente através da terapia cognitivacomportamental (TCC) que atua para aumentar a resposta farmacológica e o tempo de remissão da doença, reduzindo o período de latência do sono e do tempo acordado, melhorando a qualidade do sono. São utilizados diversos tipos TCC no tratamento da insônia como a terapia de controle de estímulos, treinamento de relaxamento, intenção paradoxal, biofeedback e higiene do sono (Neves et al., 2017).

Entretanto, a melatonina além de induzir ao sono atua como um neuroprotetor por ocasião de sua potente ação antioxidante. Age também no transtorno do jet lag, que é produzido pela dessincronizarão do relógio circadiano ao fazer uma viagem transmeridiana, minimizando os principais sintomas ocasionados pelo fuso horário como, a insônia à noite, sonolência diurna, mal-estar gastrointestinal e dores de cabeça, promovendo a ressincronização do relógio circadiano (Rubio et al., 2011).

A melatonina (Figura 1) tem sua síntese e secreção na escuridão e diminuída com a incidência da luz, uma vez que a luminosidade tem influência direta sobre a retina, que junto com os neurônios controladores da glândula pineal ditam o ritmo de sua produção através de uma via multi-sinaptica. Este controle tem alterações sazonais, visto que a duração da produção de melatonina é maior em dias curtos de inverno do que em dias longos de verão e foi observado também que até a iluminação doméstica influencia sua síntese (Esposito et al., 2019). 


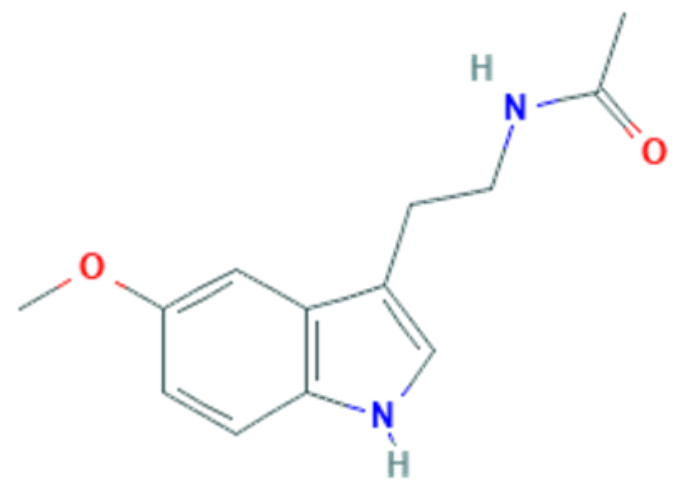

Figura 1. Estrutura química da N-acetil-5-metoxitriptamina (Melatonina). Fonte: PubChem, 2021.

O principal mecanismo de ação da melatonina, embora não esteja completamente elucidado, se dá através da ativação de dois receptores específicos de membrana (ML1 e ML2) acoplados à proteína G que levam a inibição da adenilato ciclase nas células alvo. Os receptores ML1 têm maior expressão na hipófise anterior do sistema nervoso central (SNC) no hipotálamo, onde fica localizado o relógio circadiano (Tordjman et al., 2017).

A partir do momento em que o sono comprometido traz problemas que ultrapassam a capacidade adaptativa das pessoas, causando desconforto físico e psicológico, se tornam patológicos. Pesquisas mostram que o uso de melatonina reduz o período de latência do sono, melhorando sua qualidade e que após cerca de 6 meses de tratamento não foram observados nos pacientes sintomas de abstinência, tolerância, nem insônia de rebote (Torales, 2016).

Sletten et al. (2018) realizaram um ensaio clínico randomizado em pacientes com Transtorno da Fase Vigília-Sono Retardado (DSWPD), que é caracterizado pela insônia no início do sono em horários convencionais e dificuldades em acordar no horário desejado para a realização de seus compromissos. Através do uso de melatonina combinado com o agendamento de sono-vigília foi observada uma significativa melhora clínica em relação aos distúrbios do sono e ao ritmo circadiano. Nesse estudo foi observado que após apenas uma semana os pacientes que fizeram uso de melatonina tiveram uma melhora considerável em relação aos pacientes que utilizaram o placebo.

O efeito da melatonina no organismo é de adaptação e harmonização do meio interno com o externo ajustando as funções dos órgãos através de ritmos circadianos no tocante às mudanças ambientais (Tordjman et al., 2017). Por isso foi constatado, mediante estudo, que o uso de melatonina exógena melhora a qualidade do sono alinhando-o ao ritmo circadiano (Zisapel, 2018).

Há estudos que evidenciam os benefícios da melatonina em pessoas que trabalham em ritmo de turnos variados, contribuindo para o ajuste do relógio biológico e consequentemente melhorando a qualidade do sono dessas pessoas. Existem também evidências científicas que mostram os efeitos benéficos da melatonina em pacientes com trauma de crânio e esquizofrenia, visto que, essas pessoas têm cochilos múltiplos durante o dia, alternando sono diurno com insônia noturna. A melatonina contribui de forma significativa para conciliar o ritmo monofásico do sono regulando o ciclo sono vigília (Bruin, 2015).

\section{Benzodiazepínicos e a insônia}

De acordo com a Associação Brasileira do Sono (2016), 11 milhões de brasileiros usam medicamentos para dormir, o que corresponde a 7,6\% da população. Para o tratamento farmacológico, existem três classes básicas utilizadas no tratamento da insônia: os benzodiazepínicos, os hipnóticos e os anti-histamínicos. Os benzodiazepínicos, sendo os mais utilizados, têm ação ansiolítica causando uma falsa sensação de sono, diminuindo o tempo de latência. Porém, podem causar déficit de memória e dependência (Rosa \& Borja, 2019).

Os efeitos sedativos e hipnóticos dos benzodiazepínicos ocorrem por sua atividade potencializadora sobre a ação do ácido gama-aminobutírico (GABA), o neurotransmissor inibidor primário do sistema nervoso central (SNC). Para o tratamento de insônia, não há estudos que apoiem o uso dos benzodiazepínicos por longo período por causa de sua ineficácia. Entre as prováveis explicações para isso estão a insônia de rebote, tolerância e desregulação da arquitetura do sono. Até mesmo em uso a curto prazo, os benzodiazepínicos podem diminuir o tempo do sono, o sono profundo e aumentar a latência do sono de movimento rápido dos olhos (REM) (Guina \& Merrill, 2018). 
Entre os benzodiazepínicos mais utilizados está o Clonazepam (Figura 2), que tem como produto de referência o Rivotril. Em um estudo ecológico realizado entre os anos 2009 e 2013 no estado do Rio de Janeiro sobre o consumo de Clonazepam (Rivotril), observou-se um aumento 6 vezes maior durante esse período. Entretanto, é importante ressaltar que as reações adversas mais frequentes ocorrem em 1/3 dos usuários e entre elas estão: perda de memória, déficit de atenção, sonolência, ataxia, convulsões, euforia e depressão respiratória. Além disso, delírio, psicose e depressão podem surgir como efeitos psicológicos (Zornazelli et al., 2019).

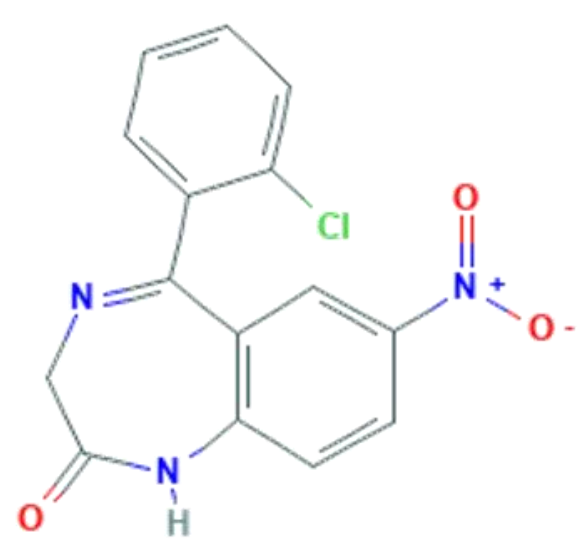

Figura 2. Estrutura química do Clonazepam. Fonte: PubChem, 2021.

De acordo com Tóth et al. (2016) sinais de abstinência, sonolência, confusão mental e tolerância são sintomas causados pelo uso de Clonazepam. Ademais, as chances de quedas e lesões em pacientes idosos aumentam com o uso deste medicamento, visto que, pode comprometer a marcha e causar distúrbios de equilíbrio. É importante salientar que a facilidade médica em receitar os benzodiazepínicos, a automedicação, a popularização entre amigos e familiares através da indicação de seu uso são fatores que estimulam o crescimento do uso irracional dessa classe de medicamentos (Firmino et al., 2011).

Embora comprovados os riscos no uso dos benzodiazepínicos, não há mudanças significativas no perfil dos usuários ao longo das décadas. As mulheres e os idosos são os principais grupos que fazem o uso inadequadamente. A aplicação das práticas integrativas e complementares, com o intuído de promover alternativas terapêuticas, pode não ter adesão aos pacientes quando os profissionais de saúde não têm conhecimento sobre a eficácia do seu tratamento e por falta de política de implantação do seu uso (Fegadolli et al., 2019).

\section{Melatonina x Clonazepam}

Apesar de serem utilizados para induzir o sono, a melatonina e o clonazepam possuem mecanismos de ação diferentes e consequentemente reações adversas distintas (Tabela 1).

Tabela 1. Comparação das reações adversas entre o Clonazepam (Rivotril) e a Melatonina.

\begin{tabular}{|c|c|c|c|}
\hline Classe farmacológica & Medicamento & Mecanismo de ação & Reações adversas \\
\hline Benzodiazepínicos & $\begin{array}{c}\text { Rivotril } \\
\text { (Clonazepam) }\end{array}$ & $\begin{array}{l}\text { Inibidor do } \text { SNC, } \\
\text { potencializando o GABA. }\end{array}$ & $\begin{array}{l}\text { Sonolência, ataxia, perda de memória, déficit } \\
\text { de atenção, euforia transitória, depressão } \\
\text { respiratória, convulsão, delírio, psicose e } \\
\text { depressão. }\end{array}$ \\
\hline Melatoninérgicos & Melatonina & $\begin{array}{l}\text { Ação nos receptores ML1 e } \\
\text { ML2, inibindo a adenilato } \\
\text { ciclase no hipotálamo. }\end{array}$ & $\begin{array}{l}\text { Sonolência diurna, possíveis dores de cabeça e } \\
\text { raros sintomas intestinais. }\end{array}$ \\
\hline
\end{tabular}

Fonte: Autor, 2021; Nakaoka et al., 2013; Tordjman et al., 2017.

Conforme tabela acima é possível perceber as diferenças nos resultados dos tratamentos contra a insônia através da melatonina e do Clonazepam. Há, portanto, a necessidade de avaliar a forma terapêutica a ser utilizada, visando um tratamento mais eficaz, não apenas tratando os sintomas, contudo, diminuindo os efeitos danosos à saúde.

Escribá e Hoyo (2016) realizaram um estudo com 23 pacientes com transtorno de comportamento do sono REM (RBD) com média de idade de 64,7 anos. Foram utilizados no estudo gabapentina, pregabalina e melatonina para serem comparados com o Clonazepam. 14 pacientes usaram gabapentina (300 e $800 \mathrm{mg}$ ) e o 
tratamento foi eficaz em 12 pessoas. 3 pacientes foram tratados com pregabalina (75 e 150mg), sendo eficaz em 2 pacientes. 5 pessoas fizeram uso de melatonina $2 \mathrm{mg}$, onde 4 participantes do estudo tiveram êxito no tratamento. 1 paciente utilizou benzodiazepínico com eficácia. Embora todas as alternativas tenham se mostrado como melhores opções ao Clonazepam, vale ressaltar que a gabapentina e a pregabalina são anticonvulsivantes e antiepiléticos e possuem elevados efeitos adversos quando comparados com a melatonina, além das doses usadas no estudo serem superiores.

Estudos de imagem do cérebro de pessoas acordadas mostraram que a melatonina tem atividade moduladora semelhante ao sono real. Foi comprovada, também, a eficácia da melatonina durante a retirada dos benzodiazepínicos, já que a descontinuação do seu uso melhora o desempenho psicomotor e cognitivo, além de contribuir para melhorar a qualidade do sono. Outro estudo realizado com 38 pacientes com doença de Parkinson mostrou que tanto a melatonina como o clonazepam reduziram os distúrbios do sono, porém o grupo que usou clonazepam teve sonolência diurna aumentada significativamente, o que não ocorreu com o grupo que fez uso da melatonina. Portanto, a melatonina pode se tornar terapia de escolha, também, para reduzir a dependência do uso de benzodiazepínicos (Cardinali et al., 2016).

\section{Considerações finais}

A insônia tem sido considerada o principal sintoma de vários distúrbios que levam a comprometer a qualidade do sono, como fatores genéticos, físicos, mentais e sociais, tornando-se assim um problema de saúde pública. $\mathrm{O}$ uso indiscriminado cada vez maior dos benzodiazepínicos tem trazido sérias complicações aos pacientes. A alternativa ao tratamento contra insônia através do uso de melatonina tem se apresentado como uma opção com menos efeitos adversos, além de atuar com outras ações benéficas no organismo, como restaurador neural e agente antioxidante. Ainda é muito restrito o uso de melatonina no Brasil devido à falta de informações sobre sua eficácia no tratamento contra insônia, apesar de haver vários estudos comprovando seus resultados. A pressão do mercado farmacêutico é outro fator que também contribui, além de ser vendida somente sob prescrição médica em farmácias de manipulação. Embora seja comercializado na Europa e EUA de forma livre como suplemento, faz-se necessário no Brasil, uma legislação através dos órgãos competentes que facilite sua aquisição, visto que, se comparada aos benzodiazepínicos possui vantagens quanto à sua eficácia e seus efeitos colaterais. Contudo, são necessários mais estudos conclusivos que esclareçam o mecanismo de ação da melatonina exógena e sua eficácia sobre os mais diversos tipos de distúrbios que comprometem a qualidade do sono.

\section{Referências}

Bacelar, A., \& Pinto Júnior, L. 2013. Insônia: do diagnóstico ao tratamento. III Consenso Brasileiro de Insônia 2013. Associação Brasileira do Sono. São Paulo, SP: Editora e Eventos Omnifarma Ltda.

Bruin, V. M. S. 2015. Importância da melatonina na regulação do sono e do ritmo circadiano: uma abordagem clínica. Sociedade Brasileira para o progresso da ciência.

Cardinali, D. P., Golombek, D. A., Rosenstein, R. E., Brusco, L. I. \& Vigo, D. E. 2016. Assessing the efficacy of melatonin to curtail benzodiazepine/Z drugabuse. Pharmacological Research, 109, 12-23.

Escribá J., \& Hoyo B. 2016. Alternatives to clonazepam in REM behavior disorder treatment. Journal of Clinical Sleep, 12(8), 1193.

Esposito, S., Laino, D., D’lonzo, R., Mencarelli, A., Di Genova, L., Fattorusso, A., Argentiero, A., \& Mancaroni, E. 2019. Pediatric sleep disturbances and treatment with melatonin. Journal of Translational Medicine, 17, 77.

Fegadolli, C. Perceptions and experiences of benzodiazepine users in primary health care in Cuba. Revista Saúde Sociedade São Paulo, 28(4), 137-146.

Firmino, K. F., Abreu, M. H. N. G., Perini, E., \& Magalhães, S. M. S. 2011. Fatores associados ao uso de benzodiazepínicos no serviço municipal de saúde da cidade de Coronel Fabriciano, Minas Gerais, Brasil. Caderno de Saúde Pública, 27(6), 1223-1232.

Frauger, E., Pauly, V., Pradel, V., Rouby, Arditti, J., Thirion, X., Mestre, M. L., \& Micallef, J. 2011. Evidence of clonazepam abuse liability: results of the tools developed by the French Centers for Evaluation and Information on Pharmacodependence (CEIP) Network. Fundamental E Clinical Phamacology, 25, 633-641. 
Glanzmann, R., Moreira, L. F., Marques, S. A., Silva, K. C., \& Soares, V. C. G. 2019. O uso da melatonina como indutor do sono: uma revisão bibliográfica. Revista Uningá, 56(1), 157-167.

Guina, J., \& Merrill, B. 2018. Benzodiazepines I: Upping the Care on Downers: The Evidence of Risks, Benefits and alternatives. Journal Clinical Medicine, 7(2), P.17.

Kalmbach, D. A., Anderson, J. R., \& Drake, C. L. 2018. The impact of stress on sleep: pathogenic sleep reactivity as a vulnerability to insomnia and circadian disorders. Journal of Sleep Research, 27(6), 12710.

Nakaoka, V. Y., Pereira, A. M. O., \& Kashiwabara, T. G. B. 2013. Qualidade do sono e melatonina: relato de caso. Brazilian Journal Of Surgery And Clinical Research, 4(1), 32-36.

Neves, G. S. M., Macedo, P. \& Gomes, M. M. 2017. Transtornos do sono: atualização (1/2). Revista Brasileira de Neurologia, 53(3), 19-30.

Quinhones, M. S., \& Gomes, M. M. 2011. Sono no envelhecimento normal e patológico: aspectos clínicos e fisiopatológicos. Revista Brasileira dee Neurologia, 47(1), 31-42.

Rosa, R. C. \& Borja, A. 2019. O uso da melatonina na insônia. Revista acadêmica Osvaldo Cruz, 6(23).

Rubio, G. J., Chagoyán. H. S., Alonso, A. D. \& King, G. B. 2011. Alteraciones del ciclo circadiano em las enfermidades psiquiátricas: papel sincronizador de la melatonina en el ciclo sueño-vigilia y la polaridad neuronal. Salud Mental, 34(2).

Silva, D. B., Gabas, D. V., Omitto, R. F., Silva, R. J. P. \& Moreno, A. H. 2020. Benefícios do uso da melatonina no tratamento da insônia e qualidade do sono. Cuidarte, Enfermagem, 14(1), 75-80.

Sletten, T. L., Magee, M., Murray, J. M., Gordon, C. J., Lovato, N., Kennaway, D. J., ... Rajaratnam, S. M. W. 2018. Efficacy of melatonin with behavioural sleepwake scheduling for delayed sleep-wake phase disorder: a double-blind, randomized clinical trial. Plos Medicine, 15(6).

Torales, J. 2016. Lo que todo médico internista debe saber acerca del manejo farmacológico del insomnio. Revista Virtual de la Sociedad Paraguaya de Medicina Interna, 3(2), 101-111.

Tordjman, S., Chokron, S., Delorme, R., Charrier, A., Bellissant, E., Jaafari, N. \& Fougerou, C. 2017. Melatonin: pharmacology, functions and therapeutic benefits. Current Neuropharmacology, 15(3), 434-443.

Tóth, K., Csukly, G., Sirok, D., Belic, A., Kiss, A., Háfra, E., ... Monostory, K. 2016. Optimization of clonazepam therapy adjusted to patient's CYP3a status and NAT2 genotype. International Journal of Neuropsychopharmacology, 19(12), 1-9.

Vyazovskiy, V. V., Walton, M., Peirson, S.N. \& Bannerman, D. M. 2017. Sleep homeostasis, habits and habituation. Current Opinion In Neurobiology, 44, 202-211.

Xie, Z., Chen, F., Li, W. A., Geng, X., Li, C. \& Meng, Xi. 2017. A Review of sleep disorders and melatonin. Neurological Research, 6, 559-565.

Zisapel, N. 2018. New perspectives on the role of melatonin in human sleep, circadian rhythms and their regulation. British Journal Of Pharmacology, 175, 3190-3199.

Zornazelli, R. T., Giordani, F., Guaraldo, L., Matos, G. C., Brito Junior, A. G., Oliveira, M. G., ... Rozenfeld, S. 2019. Consumo do benzodiazepínico clonazepam (Rivotril ${ }^{\circledR}$ ) no estado do Rio de Janeiro, Brasil-2013: estudo ecológico. Ciência e Saúde Coletiva, 24(8), 3129-3140.

\section{Minicurrículo}

Wellington Oliveira de Carvalho. Graduando do curso de Farmácia na Universidade Salvador- UNIFACS, Salvador, BA, Brasil.

Juliana Azevedo da Paixão. Farmacêutica graduada pela Universidade Federal da Bahia-UFBA, Mestre em recursos genéticos vegetais e especialista em farmacologia aplicada à prática clínica. Docente do curso de Farmácia da Universidade Salvador-UNIFACS. Salvador, BA, Brasil. 
Como citar: Carvalho, W.O., \& Paixão, J.A. 2021. Benefícios do uso de melatonina no tratamento de pessoas com distúrbios do sono, 6, 165. DOI: https://dx.doi.org/10.31533/pubsaude6.a165

Recebido: 23 abr. 2021.

Revisado e aceito: 4 mai. 2021.

Conflito de interesse: os autores declaram, em relação aos produtos e companhias descritos nesse artigo, não ter interesses associativos, comerciais, de propriedade ou financeiros que representem conflito de interesse.

Licenciamento: Este artigo é publicado na modalidade Acesso Aberto sob a licença Creative Commons Atribuição 4.0 (CC-BY 4.0). 